\title{
Accurate Measurement Setup for Strongly Inhomogeneous Magnetic Fields
}

Husstedt, Hendrik $^{1}$; Ausserlechner, Udo ${ }^{2}$; Kaltenbacher, Manfred ${ }^{1}$

${ }_{1}^{1}$ Applied Mechatronics, Alps-Adriatic University, Klagenfurt, Austria

${ }^{2}$ Sense and Control, Infineon Technologies Austria AG, Villach, Austria

Corresponding author: Hendrik Husstedt, Lakeside B04a, 9020 Klagenfurt, Austria, P: +43(0)463-2700-3568, F: +43(0)463-2700-993568, E: hendrik.husstedt@uni-klu.ac.at

Abstract - State of the art systems, measuring the spatial dependency of magnetic fields, consist of a magnetic sensor mounted in a coordinate measuring machine (CMM). A scan of the field provides roughly the components of the field vector w.r.t. the geometry of the field source. However, if strongly inhomogeneous fields are measured, small assembly tolerances of the magnetic sensor and the field source lead to crucial measurement errors. Our approach is a measurement system consisting of a CMM with magnetic sensors, and an optical sensor that measures the geometry of the field source. This combination of geometric and magnetic measurements allows for a significant reduction of assembly tolerances.

\section{Introduction}

\subsection{Motivation}

In the automobile industry, magnetic sensors are used to detect and monitor positions of throttle valves, cam- and crankshafts, pedals, wipers, winders etc. In order to characterize these sensors, dedicated measurement equipment emulates the magnetic fields during application which vary from homogeneous, to differential and rotating fields. Some of these applications require magnetic fields which are strongly inhomogeneous with a gradient up to $1 \% / 10 \mu \mathrm{m}$ (see Figure 1). To analyze magnetic measurement equipments, the spatial dependency of their field has to be measured in the volume where the device under test (DUT) is positioned.

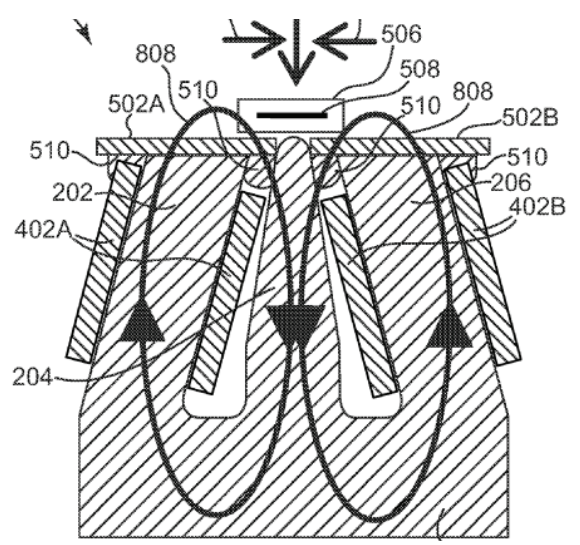

Figure 1: Schematic drawing of a measurement equipment for automotive magnetic sensors [1].

\subsection{State of the art}

State of the art systems, that can measure the spatial dependency of magnetic fields, mount a magnetic sensor in a coordinate measuring machine (CMM) which consists of several moving axis, e.g. [2]. In such a measurement system, a magnetic sensor is positioned at several points where the magnetic field is measured. Such a scan provides roughly the field vector w.r.t. the geometry of the field source. However, if the fields are strongly inhomogeneous, small assembly tolerances of the magnetic sensor and the field source lead to crucial measurement errors. Experience shows that typical errors of the position of the magnetic sensors w.r.t. the field source are $\pm 300 \mu \mathrm{m}$, and errors of the alignment are $\pm 3^{\circ}$. It is obvious that a magnetic field with a gradient of $1 \% / 10 \mu \mathrm{m}$ cannot be measured accurately with such tolerances. 


\subsection{Approach}

Our measurement system consists of a CMM with magnetic sensors and an optical sensor for geometrical measurements (see Figure 2). First, the optical sensor measures the geometry of the field source so that its exact position and alignment in the coordinate system of the CMM is known. Now, the magnetic sensor can scan the magnetic field at points relative to the field source. Finally, the measured field vectors can be transformed to the coordinate system of the field source so that assembly tolerances have no influence any more.
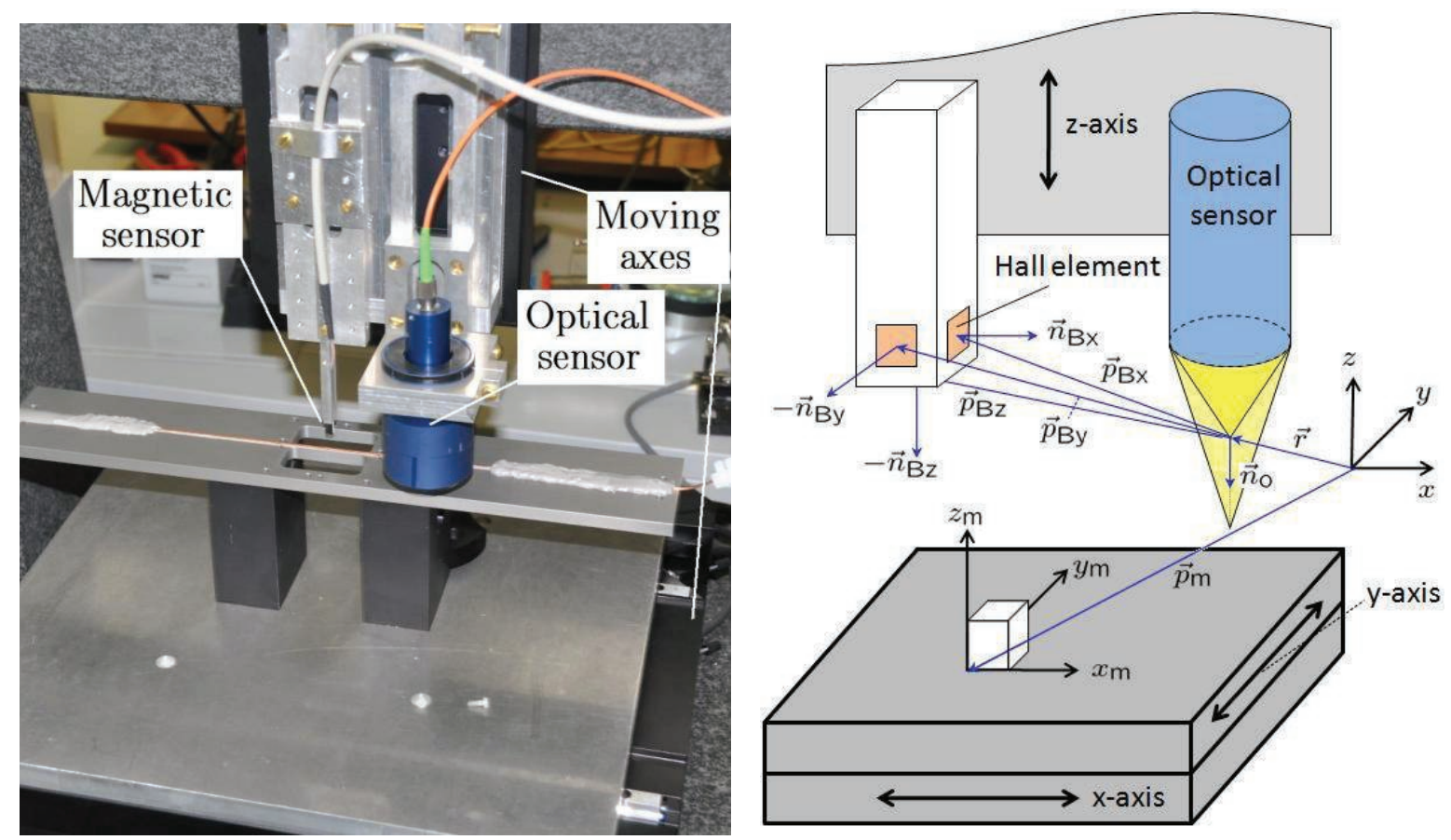

Figure 2: On the left hand side there is photograph of the measurement system consisting of a CMM, a magnetic and an optical sensor. On the right hand side, the setup is schematically visualized with the reference axes of the CMM $x, y, z$, and the coordinate system of the measurement object $x_{m}, y_{m}, z_{m}$.

\section{Calibration}

\section{$2.1 \quad$ Parameters}

Moving the magnetic sensor to points relative to the field source requires that the distance between the magnetic and optical sensor is known. Moreover, the measured field vectors can only be transformed to the coordinate system of the field source, if the alignment of the magnetic sensor w.r.t. the coordinate system of the CMM is known. Therefore, a calibration of the position and alignment of the magnetic sensor in the setup is necessary.

The measurement of all three magnetic field components requires at least three one dimensional sensing elements. Hall probes are chosen, because they are sensitive to magnetic fields used in automotive applications $(10 \mu \mathrm{T}-200 \mathrm{mT})$, and they have a linear characteristic without hysteresis. State of the art integrated Hall sensors may have several sensing elements on one silicon die and measure the magnetic induction in one [3], two [4] or all three directions [5, 6]. The calibration of one sensing element requires the measurement of three degrees of freedom for the position vector $\vec{p}_{B}$ and two degrees of freedom for the alignment $\vec{n}_{B}$ (see right part of Figure 2). So, in general, 15 degrees of freedom have to be calibrated for all three directions $\left(\vec{p}_{B i}, \vec{n}_{B i}\right.$ with $\left.i \in\{x, y, z\}\right)$. However, if a multidimensional sensor is assembled, and the alignment and position of the sensing elements are exactly known relative to each other, not all distances and normal vectors have to be measured.

Beside the calibration of assembly tolerances, the main parasitic effects of the Hall probes such as offset and sensitivity drift have to be considered. The offset is simply measured without any field source whereas the calibration of the sensitivity $S_{i}$ requires an external reference field. 


\subsection{Method}

For the calibration of the position, alignment, and sensitivity of the magnetic sensing elements, an inhomogeneous reference field source is used which has a strong relation between its magnetic field and its geometry. Moreover, the magnetic reference field $\overrightarrow{\mathrm{B}}_{\mathrm{r}}(x, y, z)=\overrightarrow{\mathrm{B}}_{\mathrm{r}}(\vec{r})$ can be described analytically. First, the optical sensor measures the position and alignment of the field source so that the magnetic reference field is known. With this information the output signal $M_{i}$ of any sensing element can be calculated as a function of the sensitivity $S_{i}$, the alignment $\vec{n}_{B i}$, and the position $\vec{p}_{B i}$

$$
M_{i}=S_{i} \vec{n}_{B i} \cdot \vec{B}_{r}\left(\vec{r}-\vec{p}_{B i}\right) .
$$

Then, the magnetic sensor scans the field which results in the output signal $\widetilde{M}_{i}$. Finally, an optimization problem is formulated where the mean squared error between the calculated and measured values should be minimized

$$
\min _{S_{i}, \overrightarrow{\tilde{n}}_{B i}, \vec{p}_{B i}}\left\|\widetilde{M}_{i}-M_{i}\right\|^{2}
$$

Solving this minimization problem results in the unknown parameters of calibration.

\section{$2.3 \quad$ Reference field}

The reference field is provided by a current supplied, straight conductor with a circular cross section. If all materials are non permeable and the conductor is assumed to be infinite, the magnetic induction outside of the conductor is given by

$$
\vec{B}_{c}(a)=\frac{\mu_{0} I}{2 \pi a} \vec{e}_{\varphi}
$$

where $a$ denotes the orthogonal distance to the axis of the conductor, and $\vec{e}_{\varphi}$ the tangential unit vector w.r.t. the axis of the conductor [7]. In the coordinate system of the CMM the axis of the conductor is defined by the linear equation

$$
\vec{g}_{c}(\lambda)=\vec{p}_{c}+\lambda \vec{d}_{c}
$$

where $\vec{p}_{c}$ is the position vector and $\vec{d}_{c}$ the direction vector. With this description, the magnetic field of the conductor is represented by

$$
\vec{B}_{r}(\vec{r})=\frac{\mu_{0} I}{2 \pi}\left|\vec{d}_{c}\right| \frac{\vec{d}_{c} \times\left(\vec{r}-\vec{p}_{c}\right)}{\left|\vec{d}_{c} \times\left(\vec{r}-\vec{p}_{c}\right)\right|^{2}} .
$$

\subsection{Generating the reference field}

To generate the reference field, a conductor made of copper is stretched and then glued to a frame made of aluminum (see Figure 3 ). The stretching supports the conductor axis to be straight and the cross section to be circular. On the frame, the position of the conductor is defined by a small guiding channel which is filled with adhesive. At the ends, the conductor is completely covered with adhesive, since the connection of the conductor to the power supply results in stress in these areas. Moreover, the adhesive, Loctite Hysol 9497, is optimized for metallic surfaces and has a high thermal conductivity which is important for heat dissipation from the conductor to the attachment. The frame is anodized to electrically isolate the conductor from the aluminum attachment so that it is surrounded by a hard coating. In the center there are two openings so that the magnetic sensor can be positioned over and beside the conductor. Moreover, the frame can be attached with fixing pins and reamed holes in the measurement setup in several orientations (see Figure 4). Finally, optical measurements of the conductor surface in the range of $\pm 70 \mathrm{~mm}$ around the center show that the error of the geometry compared to an ideal cylinder is in the range of $\pm 10 \mu \mathrm{m}$. 


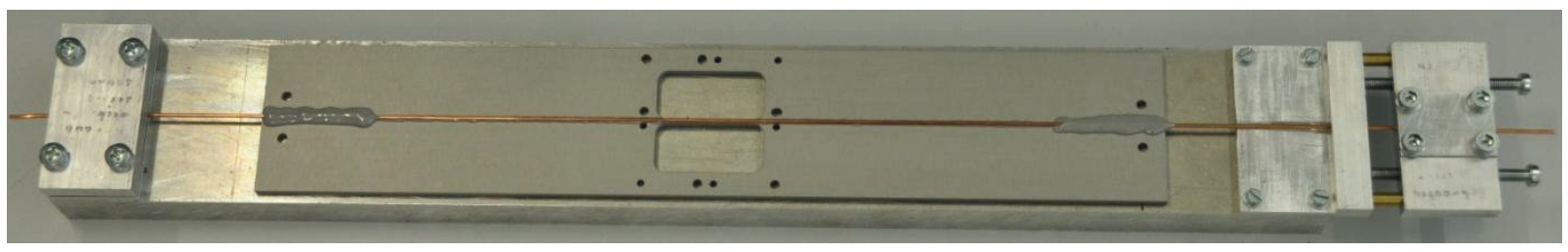

Figure 3: Conductor while it is stretched and glued to the aluminum frame.

\subsection{Orientation of the conductor}

The orientation of the conductor has to fit to the orientation of the Hall plate to assure an adequate measurement signal. If the axis of the conductor and the normal vector of the Hall plate are in parallel no measurement signal occurs. Secondly, the surface of the conductor has to be measured optically, thus, an orientation of the conductor parallel to the z-axis of the CMM is not possible. Thirdly, the conductor is homogeneous along its rotation axis, and a measurement in one orientation restricts the position of the Hall plate only to a line parallel to the conductor axis. Therefore, at least two measurements with different orientation of the conductor are necessary. Finally, after all considerations, four orientations of the conductor are enough to calibrate all three Hall plates (see Figure 4).
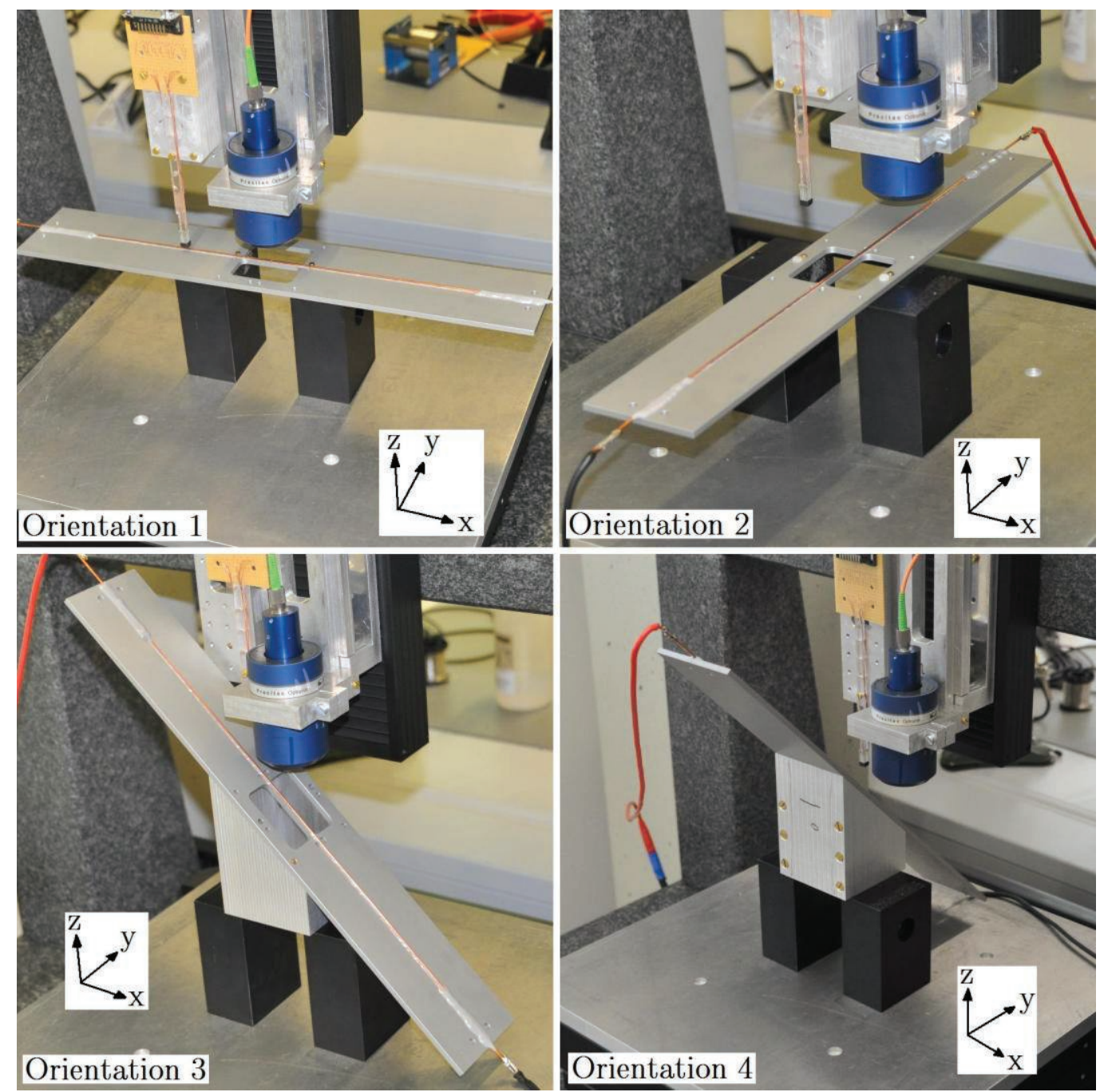

Figure 4: Possible orientations of the conductor in the CMM reference frame.

\subsection{Realization}

The calibration of one Hall plate requires that the conductor is attached in two orientations. For each orientation the geometry is measured with the optical sensor first so that the position vector $\vec{p}_{c}$ and the direction vector $\vec{d}_{c}$ is known, and the magnetic reference field according to ( 4 ) can be calculated. 
Then, a scan with the Hall plate around the conductor is performed and the optimization problem ( 2 ) is formulated. Finally, solving this problem leads to the desired calibration parameters such as the position $\vec{p}_{B}$, the alignment $\vec{n}_{B}$, and the sensitivity $S$.

\section{Accuracy assumption}

Firstly, the length of the conductor cannot be infinite. If the length is limited, the strength of the magnetic field is reduced, but the direction does not change [7]. During the measurement, the distance of the Hall plate to the axis of the conductor is small compared to the length of the conductor, and the reduction of the magnetic field is around $-0.01 \%$.

Secondly, the conductor is supplied with an appropriate current so that effects caused by a heating-up of the conductor can be neglected. Moreover, at each measurement point, the conductor is supplied with a positive and negative current. For this differential measurement, the output is the average of the measurement value at positive current and the negative measurement value at negative current. This calculation filters out all offsets caused by the magnetic sensor or external fields. It should be mentioned that all even terms of a nonlinear sensor characteristic are also canceled by this differential technique, even though the characteristic of Hall probes is sufficiently linear within the field range applied during calibration.

The supply cables and the coupling of the reference field with permeable material around the setup generate an interference field at the position of the sensor which is not eliminated by the differential measurement technique. Even though all attachments, screws etc. are made of non magnetic materials, there are some parts in the moving system such as the guide rails which are permeable. Both the supply cables and the permeable material are far away compared to the distance between the Hall plate and the conductor. Therefore, the interference field is almost homogenous at the position of the magnetic sensor and can be approximated by a constant term. This term is additionally fitted

$$
M_{i}=S_{i} \vec{n}_{B i} \cdot \vec{B}_{r}\left(\vec{r}-\vec{p}_{B i}\right)+M_{\text {offset }}
$$

so that the impact of the supply cables and permeable materials is sufficiently suppressed.

Finally, all sources of errors are combined in one model. To this end, the magnetic fields of the conductor and the supply cables are calculated by means of the Biot Savart law. Moreover, in the range of $\pm 70 \mathrm{~mm}$ the axis of the conductor is represented by the data measured by the optical sensor which considers the tolerances of the conductor geometry. Averaging the magnetic field over several points around the center of the sensor takes the finite size of the Hall element into account. Finally, noise is added to the averaged measurement signal and a Monte Carlo simulation is performed which results in probability distributions of the calibration parameters (position, alignment, and sensitivity). With the results of the model, the accuracy of the $\mathrm{CMM}$, and a temperature stability of $0.5^{\circ} \mathrm{C}$, the accuracy of the setup is estimated to be $10 \mu \mathrm{m}$ for the position, $0.1^{\circ}$ for the alignment, and $0.4 \%$ for the sensitivity. Moreover, if a higher accuracy for the sensitivity is needed, the sensor can be calibrated in a Helmholtz coil, and a higher accuracy for the alignment can be reached by using the calibration algorithm explained in [8].

\section{$4 \quad$ Summary}

A measurement system is shown that consists of a moving system with an optical and magnetic sensor. The combination of both sensors allows for measurements of the magnetic fields w.r.t. the geometry of the field source. To this end, a calibration of the position and alignment of the magnetic sensor is necessary. During the calibration the magnetic field of a current supplied straight conductor is used as reference. Furthermore, it is presented how the reference field is generated, and the accuracy of the calibration is estimated. To conclude, the presented setup is a powerful tool to analyze strongly inhomogeneous magnetic fields. 


\section{References}

[1] U. Ausserlechner and M. Holliber, U.S. Patent Application US 2009/0066465 A1, 2009.

[2] XYZ Field Scanner, Dr. Brockhaus Messtechnik GmbH \& Co. KG, Gustav-Adolf-Str. 4, 58507 Lüdenscheid, Germany.

[3] TLE4998S3C: Linear Hall sensor, Data Sheet Rev1.0, Infineon Technologies AG, Am Campeon 1-12, 85579 Neubiberg, Germany, 2009.

[4] 2SA-10 Two-axis, General Purpose $X / Y$, Sentron AG, Baarermattstrasse 10, CH-6300 Zug, Switzerland.

[5] H. Hohe, M. Hackner, and M. Stahl-Offergeld, "Magnetic 3d point sensor capable of being calibrated during measurement operation," German Patent WO/2008/017348, 2008.

[6] D. Popovic, S. Dimitrijevic, M. Blagojevic, P. Kejik, E. Schurig, and R. Popovic, "Three-axis teslameter with integrated hall probe," IEEE Transactions on Instrumentation and Measurement, vol. 56, pp. 1396-1402, August 2007.

[7] H. Hofmann, Das elektromagnetische Feld. Springer-Verlag, 1974.

[8] H. Husstedt, U. Ausserlechner, and M. Kaltenbacher, "Precise alignment of a magnetic sensor in a coordinate measuring machine," Sensors Journal, IEEE, vol. 10, no. 5, pp. 984 -990, May 2010. 